\title{
A new PCR followed by MboI digestion for the detection of all variants of the Clostridium perfringens cpb2 gene
}

\author{
Alphons J.A.M. van Asten ${ }^{\mathrm{a}, *}$, Janneke G. Allaart ${ }^{\mathrm{a}}$, Arjan D. Meeles ${ }^{\mathrm{a}}$, \\ Peggy W.J.M. Gloudemans ${ }^{\mathrm{a}}$, Dirk J. Houwers ${ }^{\mathrm{b}}$, Andrea Gröne ${ }^{\mathrm{a}}$ \\ ${ }^{a}$ Department of Pathobiology, Faculty of Veterinary Medicine, Utrecht University, Yalelaan 1, P.O. Box 80.158, \\ 3508 TD Utrecht, The Netherlands \\ ${ }^{\mathrm{b}}$ Veterinary Microbiological Diagnostic Center, Division of Clinical Infectiology, Department of Infectious Diseases and Immunology, Faculty \\ of Veterinary Medicine, Utrecht University, P.O. Box 80.165, 3508 TD Utrecht, The Netherlands
}

Received 31 May 2007; received in revised form 16 August 2007; accepted 17 August 2007

\begin{abstract}
Clostridium perfringens which is a causative agent of several diseases in animals and humans is capable of producing a variety of toxins. Isolates are typed into five types on the basis of the presence of one or more of the four major toxins genes, i.e. $c p a, c p b$, etx, and iap. A decade ago another toxin termed beta2 ( $\beta 2)$ and its gene ( $c p b 2)$ were identified. Two alleles of $c p b 2$ are known and a possible link between differences in gene expression and allelic variation has been reported. A correlation between the level of expression and the origin of the isolates has also been suggested. The demonstration and typing of the $c p b 2$ gene in the genome of isolates can be seen as a vital part of research on the role of the beta 2 toxin in the pathogenesis of disease. This study describes a PCR with a single primer set which in contrast to published primer sets recognizes both alleles. Subsequent restriction enzyme analysis of the PCR product enables typing of the alleles. Applying this protocol on a total of 102 isolates, a sub-variant was found which occurred only in $C$. perfringens isolates from pigs and appeared to be the predominant variant found in $C$. perfringens isolates from this species.
\end{abstract}

(C) 2007 Elsevier B.V. All rights reserved.

Keywords: Clostridium perfringens; cpb2; Beta2 toxin; PCR

\section{Introduction}

The Gram-positive anaerobic bacterium Clostridium perfringens is part of the normal intestinal flora and is

\footnotetext{
* Corresponding author. Tel.: +31 302534307; fax: +31302516853 .

E-mail address: f.asten@vet.uu.nl (A.J.A.M. van Asten).
}

ubiquitous in the environment. However, it is also an important cause of enteric diseases in animals and humans. Type and severity of the disease are correlated with the toxin(s) produced by the bacterium (Songer, 1996). Based on the four major toxins (alpha, beta, epsilon and iota) the strains of $C$. perfringens are classified into five toxin types: types A-E, e.g. by demonstrating the presence of the encoding genes by PCR. 
Another toxin produced by a $C$. perfringens strain was described a decade ago (Gibert et al., 1997). This toxin was designated beta2 since its biological activities were comparable to those of the beta toxin. The gene encoding the beta 2 toxin was named $c p b 2$ and shown to be located on a plasmid (Gibert et al., 1997). The $c p b 2$ gene has been demonstrated in isolates originating from a variety of animals, i.e. pigs, horses (Gibert et al., 1997), lambs, calves (Garmory et al., 2000), chickens (Siragusa et al., 2006), dogs (Thiede et al., 2001), and from humans (Fisher et al., 2005). It was shown that $88.5 \%$ of the $c p b 2$-positive nonporcine isolates $(n=78)$ and 2 out of $76(2.6 \%)$ cpb2-positive porcine isolates examined carried a variant of $c p b 2$. This variant had a sequence-identity of only 70.2-70.7\% with the original $c p b 2$ (GenBank accession number L77965) resulting in a 62.3\% identity and a $80.4 \%$ similarity at amino-acid level (Jost et al., 2005). The original cpb2 was termed the consensus gene/allele whereas the variant was termed the atypical gene or atypical allele (Jost et al., 2005, 2006). Up to now all known $c p b 2$ sequences can be assigned to one of the two variants with some ambiguities within each variant. In order to demonstrate the presence of $c p b 2$, several sets of primers have been used. However, using BLAST (Basic Local
Alignment Search Tool; http://www.ncbi.nlm.nih.gov/ BLAST/) none of the published primer combinations recognized all $c p b 2$ sequences in the Genbank/EMBL/ DDBJ databases. The aim of this study was to design primers recognizing all available $c p b 2$ sequences and to find ways to discriminate between the PCR products originating from either the atypical or the consensus gene (allele).

\section{Materials and methods}

\subsection{Bacteria}

Sixty $C$. perfringens strains used in the development of the PCR were from the collection of the Veterinary Microbiological Diagnostic Center (VMDC), Division of Clinical Infectiology, Department of Infectious Diseases and Immunology, Faculty of Veterinary Medicine, Utrecht University. Fecal samples used in the evaluation of the protocol for the demonstration and typing of $C$. perfringens in clinical samples originated from pigs, sheep, cows, horses, dogs and cats as well as from a variety of other (exotic) species. Isolation of $C$. perfringens from these samples was performed using standard procedures.

Table 1

Primers used

\begin{tabular}{|c|c|c|c|c|c|}
\hline Name prime & Target gene & Sequence (5'-3') & $\mathrm{T}_{\mathrm{an}}$ & Product length & Name primer set \\
\hline Cpbeta2 $\mathrm{F}^{\mathrm{a}}$ & Consensus $c p b 2$ & CAAGCAATTGGGGGAGTTTA & & $200 \mathrm{bp}$ & Consensus-primer set \\
\hline Cpbeta2R ${ }^{a}$ & Consensus $c p b 2$ & GCAGAATCAGGATTTTGACCA & $53^{\circ} \mathrm{C}$ & & \\
\hline CpEEB2F ${ }^{b}$ & Atypical $c p b 2$ & AACATAATAAATCCTATAACCC & $50^{\circ} \mathrm{C}$ & $1239 \mathrm{bp}$ & Atypical-primer set \\
\hline CpEEB2R ${ }^{b}$ & Atypical $c p b 2$ & ATAAATATAATTCTCTAAAACC & $50^{\circ} \mathrm{C}$ & & \\
\hline CpB2totalF2 ${ }^{c}$ & Consensus/atypical $c p b 2$ & AAATATGATCCTAACCAAM ${ }^{d}$ AA & $48^{\circ} \mathrm{C}$ & $548 \mathrm{bp}$ & Total-primer set \\
\hline CpB2totalR ${ }^{c}$ & Consensus/atypical $c p b 2$ & CCAAATACTY ${ }^{\ominus}$ TAATYGATGC & $48^{\circ} \mathrm{C}$ & & \\
\hline CPAF $^{\mathrm{f}}$ & Cpa & GCTAATGTTACTGCCGTTGA & $53^{\circ} \mathrm{C}$ & $325 \mathrm{bp}$ & CPA-primer set \\
\hline CPAR $^{f}$ & Cpa & ССTCTGATACATCGTGTAAG & $53^{\circ} \mathrm{C}$ & & \\
\hline
\end{tabular}

${ }^{\mathrm{a} B a u m s}$ et al. (2004).

${ }^{b}$ Jost et al. (2005).

${ }^{\mathrm{c}}$ This paper.

${ }^{\mathrm{d}} \mathrm{M}=\mathrm{A}$ or $\mathrm{C}$.

${ }^{\mathrm{e}} \mathrm{Y}=\mathrm{C}$ or $\mathrm{T}$.

${ }^{\mathrm{f}}$ Meer and Songer (1997). 


\subsection{Primers and PCR conditions}

Primers used are given in Table 1. For sample preparation one $C$. perfringens colony was resuspended in $25-50 \mu l$ of distilled water and heated for 10 min at $95{ }^{\circ} \mathrm{C}$. Fifty microliters of PCR reaction mix consisted of $1 \mu \mathrm{l}$ of this heated bacterial suspension, $100 \mathrm{ng}$ of each primer (Isogen, The Netherlands), $0.2 \mathrm{mM}$ of each dNTP (Fermentas, Germany), $1 \mathrm{U}$ recombinant $T a q$ DNA polymerase (Fermentas), $5 \mu \mathrm{l}$ of $10 \times \mathrm{Taq}$ buffer, $1.5 \mathrm{mM} \mathrm{MgCl}_{2}$ and distilled water. The PCR program consisted of $3 \mathrm{~min}$ at $95^{\circ} \mathrm{C}$ followed by 40 cycles of $30 \mathrm{~s}$ at $95{ }^{\circ} \mathrm{C}, 30 \mathrm{~s}$ at the annealing temperature (Table 1) and amplification at $72{ }^{\circ} \mathrm{C}$ during the appropriate time $(1 \mathrm{~kb} / \mathrm{min})$. A final amplification step of $7 \mathrm{~min}$ at $72{ }^{\circ} \mathrm{C}$ completed the PCR reaction. PCR was performed using either the GeneAmp PCR system 2400 (Perkin Elmer, USA) or the iCycler (BioRad, England). As a sample preparation control, all samples were subjected to a PCR for the cpa gene which is present in all $C$. perfringens strains (Titball et al., 1999). After performing the PCR reaction, the total reaction volume was subjected to gel electrophoresis.

\subsection{Sequence and restriction enzyme analysis}

PCR products were extracted from gel using the QIAEX II Gel Extraction Kit (Qiagen) and subsequently sequenced (BaseClear, The Netherlands). The resulting sequences were mapped for restriction sites using the NEBcutter software (www.neb.com).

\section{Results}

\subsection{PCR}

Sixty strains of the collection of the VMDC were tested with the consensus-primer set, the atypicalprimer set, and the new total-primer set for the presence of the $c p b 2$ gene. A total of 43 strains were negative with all primer sets. Eight strains were positive with the consensus-primer set but negative with the atypicalprimer set. Nine strains were positive with the atypicalprimer set but negative with the consensus-primer set. However, these seventeen strains were all positive with the newly designed total-primer set.

\subsection{Sequence results and restriction enzyme analysis}

A PCR product of each variant generated by the total-primer set was sequenced. Alignment of the sequence obtained from the consensus primers positive strain (accession number EU085382) using the BLAST software showed a $98-100 \%$ identity with known sequences of this variant. Analyzing the sequence of the PCR product from the atypicalprimer set positive strain (accession number EU085383) resulted in a 94-99\% identity with the available sequences of the atypical variant. Next, both sequences were subjected to restriction enzyme analysis which showed that digestion with the enzyme MboI would result in two clearly different restriction patterns; the consensus strain product would result in fragments with sizes of, respectively, 6, 114, 164 and $264 \mathrm{bp}$ whereas the atypical strain product would generate two $6 \mathrm{bp}$ fragments, one fragment of $193 \mathrm{bp}$ and one of $343 \mathrm{bp}$. Subsequently all PCR products obtained with the total-primer set were purified from gel using the QIAEX II kit (Qiagen) and digested with (FastDigest) MboI (Fermentas) according to the manufacturers instructions. Indeed MboI digestion and subsequent electrophoresis of all nine PCR products from the atypical-primer set positive strains showed bands of the predicted sizes (Fig. 1). However, MboI digestion of only four out of the eight PCR products from consensus-primer set positive strains resulted in fragments of the expected sizes (con3 pattern, Fig. 1). The other four PCR products showed only two visible bands: one band of $164 \mathrm{bp}$ and one of approximately $390 \mathrm{bp}$ (con2 pattern, Fig. 1). Partly sequencing of one of these PCR products (accession number EU085384) revealed a difference in nucleotide 270 (TATC versus GATC) between this sequence and seq EU085382 resulting in a difference in the number of MboI restriction sites. Indeed in silica restriction analysis indicated three fragments: one $6 \mathrm{bp}$ fragment, one fragment of $164 \mathrm{bp}$ and one fragment of $378 \mathrm{bp}$. Strikingly the PCR products with a con 2 pattern were generated from strains originating from pigs whereas the PCR products resulting in one of the two other MboI patterns were from nonporcine origin. In order to study the possible correlation between MboI pattern and allelic variant as well as the possible correlation between MboI pattern and sample 


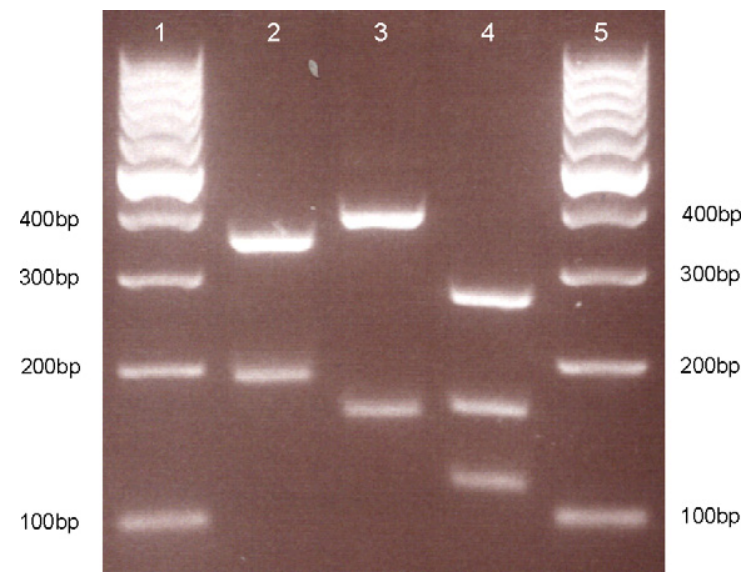

Fig. 1. Agarose gel (3\%) electrophoresis of MboI digests of PCR products obtained with the total-primer set on an atypical sample (lane 2) or consensus samples (lanes 3 and 4). Lanes 1 and 5: size marker (MassRuler Express DNA ladder Mix, Fermentas). The presence of the 6 bp bands cannot be demonstrated in this gel under given conditions.

origin, 36 atypical sequences and $37 c p b 2$ consensus sequences in the Genbank/EMBL/DDBJ databases were analyzed for the MboI restriction pattern. The atypical sequences all originating from nonporcine sources showed the predicted atypical pattern. Analysis of 24 consensus sequences originating from sheep $(n=4)$, cattle $(n=7)$, horses $(n=6)$, humans $(n=5)$ and other sources $(n=2)$ resulted in the con3 pattern. Thirteen consensus sequences all originating from pig isolates (including the firstly described $c p b 2$ gene with accession number L77965) resulted in the con2 pattern. Analyzing 19 submitted partial consensus sequences showed that 17 harbored the MboI site at position 270 downstream the first nucleotide of the CpB2total F2 primer. These sequences all originated from nonporcine sources like food, poultry, etc., whereas two sequences which were devoid of this MboI site were of porcine origin.

\subsection{Use of the new total-primer set and MboI digestion in routine screening}

Next, a protocol for the demonstration and typing of the $c p b 2$ containing $C$. perfringens in clinical samples was formulated. At the VMDC, probable $C$. perfringens colonies from clinical samples were streaked on blood agar for single colonies. Subse- quently, one colony was used in a PCR with the new total-primer set and a PCR with the CPA-primer set. PCR products obtained with the total-primers were digested with FastDigest MboI (Fermentas) without any purification and the resulting digest was electrophoresed on a 3\% agarose gel. Forty-two isolates were typed accordingly: all were cpa positive, 24 were positive for $c p b 2$. Mbol digestion patterns of PCR products obtained from isolates from all types of animals other than pig resulted in either the atypical pattern (17) or in the con3 pattern (5). Both products amplified from isolates from pigs showed a con 2 pattern. To confirm these findings all positive strains were also tested with the corresponding primer set. Results with the consensusprimer set were in agreement with the MboI restriction patterns. However, three isolates which were typed atypical with the total-primer set did not result in a PCR product when using the atypicalprimer set and the advised protocol (Jost et al., 2005). Changing PCR conditions like annealing temperature or annealing time did result in a PCR product from only one of these isolates. The remaining two isolates were also negative with the consensus-primer set.

\section{Discussion}

The difference in pathogenicity between $C$. perfringens strains is thought to be largely correlated with the toxins produced. The role of some of these toxins in the pathogenicity of disease has not been clarified yet. One of the toxins with an unresolved role is the beta2 toxin. C. perfringens strains harboring $c p b 2$ have frequently been isolated from or associated with enteritis in pigs (Gibert et al., 1997; Johansson et al., 2006), enterocolitis in horses (Gibert et al., 1997; Johansson et al., 2006), enterotoxaemia in calves (Lebrun et al., 2007) and diarrhea in dogs (Thiede et al., 2001). The actual presence of the toxin in affected tissue suggesting an association between the beta 2 toxin and the post-mortem findings has been demonstrated by immunohistochemistry in an African elephant (Loxodonta africana) with ulcerative enteritis (Bacciarini et al., 2001) and in horses that died from typhlocolitis (Bacciarini et al., 2003; Vilei et al., 2005). 
In order to study the role of the beta 2 toxin in various animals, a rapid screening method for the presence of the beta2 toxin would be helpful. Although the presence of $c p b 2$ in the genome of the bacterium does not necessarily mean that the toxin is produced, a PCR for the toxin gene can be seen as a first screening.

Several sets of primers for the demonstration of $c p b 2$ have been published, however when using the BLAST software none of these primers (fully) aligned with all complete $c p b 2$ sequences in the Genbank/EMBL/DDBJ databases. Therefore, primers that recognize all known (complete) $c p b 2$ sequences were designed and tested.

Recent studies indicate that there might be a difference in the toxin production between the consensus and atypical variants (Bueschel et al., 2003; Jost et al., 2005; Lebrun et al., 2007). Furthermore, the level of expression of the consensus $c p b 2$ gene seems to correlate with the origin of the isolates, with the genotype-phenotype correlation being the highest in porcine isolates (Bueschel et al., 2003; Jost et al., 2005; Vilei et al., 2005). The MboI restriction pattern of the PCR product generated with our total-primer set discriminates between atypical and consensus variants and between porcine and nonporcine origin and might therefore correlate with the level of beta2 toxin production.

In conclusion, the described combination of a PCR using the newly designed total-primer set with subsequent MboI digestion is a practical tool in the diagnostics, and may be of value in the research on the role of the beta2 toxin. Research should focus on the correlation between $c p b 2$ type and beta2 toxin production.

\section{Acknowledgements}

G. Nicolau and the technicians of the VMDC are greatly acknowledged for their skilful technical assistance.

\section{References}

Bacciarini, L.N., Pagan, O., Frey, J., Gröne, A., 2001. Clostridium perfringens $\beta 2$-toxin in an African elephant (Loxodonta africana) with ulcerative enteritis. Vet. Rec. 149, 618-620.
Bacciarini, L.N., Boerlin, P., Straub, R., Frey, J., Gröne, A., 2003. Immunohistochemical localization of Clostridium perfringens $\beta 2$-toxin in the gastrointestinal tract of horses. Vet. Pathol. 40, 376-381.

Baums, C.G., Schotte, U., Amtdberg, G., Goethe, R., 2004. Diagnostic multiplex PCR for toxin genotyping of Clostridium perfringens isolates. Vet. Microbiol. 100, 11-16.

Bueschel, D.M., Jost, B.H., Billington, S.J., Trinh, H.T., Songer, J.G., 2003. Prevalence of $c p b 2$ encoding beta2 toxin, in Clostridium perfringens field isolates: correlation of genotype with phenotype. Vet. Microbiol. 94, 121-129.

Fisher, D.J., Miyamoto, K., Harrison, B., Akimoto, S., Sarker, M.R., McClane, B.A., 2005. Association of beta2 toxin production with Clostridium perfringens type A human gastrointestinal disease isolates carrying a plasmid enterotoxin gene. Mol. Microbiol. 56, 747-762.

Garmory, H.S., Chanter, N., French, N.P., Bueschel, D., Songer, J.G., Titball, R.W., 2000. Occurrence of Clostridium perfringens beta2-toxin among animals, determined using genotyping and subtyping PCR assays. Epidemiol. Infect. 124, 61-67.

Gibert, M., Jolivet-Reynaud, C., Popoff, M.R., 1997. Beta2 toxin a novel toxin produced by Clostridium perfringens. Gene 203, $65-73$.

Johansson, A., Aspan, A., Bagge, E., Båverud, V., Engström, B.E., Johansson, K.-E., 2006. Genetic diversity of Clostridium perfringens type A isolates from animals, food poisoning outbreaks and sludge. BMC Microbiol. 6, 47.

Jost, B.H., Billington, S.J., Trinh, H.T., Bueschel, D.M., Songer, J.H., 2005. Atypical $c p b 2$ genes, encoding beta2-toxin in Clostridium perfringens isolates of nonporcine origin. Infect. Immun. 73, 652-656.

Jost, B.H., Billington, S.J., Trinh, H.T., Songer, J.G., 2006. Association of genes encoding beta2 toxin and a collagen binding protein in Clostridium perfringens isolates of porcine origin. Vet. Microbiol. 115, 173-182.

Lebrun, M., Filée, P., Mousset, B., Desmecht, D., Galleni, M., Mainil, J.G., Linden, A., 2007. The expression of Clostridium perfringens consensus beta 2 toxin is associated with bovine enterotoxaemia syndrome. Vet. Microbiol. 120, 151-157.

Meer, R.R., Songer, J.G., 1997. Multiplex polymerase chain reaction assay for genotyping Clostridium perfringens. Am. J. Vet. Res. 58, 702-705.

Siragusa, G.R., Danyluk, M.D., Hiett, K.L., Wise, M.G., Craven, S.E., 2006. Molecular subtyping of poultry-associated type A Clostridium perfringens isolates by repetitive-element PCR. J. Clin. Microbiol. 44, 1065-1073.

Songer, J.G., 1996. Clostridial enteric diseases of domestic animals. Clin. Microbiol. Rev. 9, 216-234.

Thiede, S., Goethe, R., Amtsberg, G., 2001. Prevalence of $\beta 2$ toxin gene of Clostridium perfringens type A from diarrhoeic dogs. Vet. Rec. 149, 273-274.

Titball, R.W., Naylor, C.E., Basak, A.K., 1999. The Clostridium perfringens $\alpha$-toxin. Anaerobe 5, 51-64.

Vilei, E.M., Schlatter, Y., Perreten, V., Straub, R., Popoff, M.R., Gibert, M., Gröne, A., Frey, J., 2005. Antibiotic-induced expression of a cryptic $c p b 2$ gene in equine $\beta 2$-toxigenic $C$. perfringens. Mol. Microbiol. 57, 1570-1581. 